\title{
Networked Learning Dynamics in Online Instruction: An Action Research Study
}

Carlos Sanchez-Lozano ${ }^{1, *}$

${ }^{1}$ Cenfotec University, Costa Rica

\begin{abstract}
This paper describes a research project in progress which focuses on the use of sequential analysis to identify student strategies and cognitive load within a networked learning model adopted in an online postgraduate course. Regarding the network design, epistemic, social and set aspects have been considered in the course structure and are briefly presented here. In particular, it is hypothesized that individual sequences can be related to student learning outcomes and cognitive load. The design and delivery of the course will be analyzed using an action research approach complemented by data analytics techniques using PRom and Python.
\end{abstract}

Keywords: eLearning, networked learning, sequence analysis

\section{Introduction}

E-Learning courses use a variety of instructional tools to improve student learning outcomes. Considered as a subset of broader forms of learning ecosystems or networks, they combine text-based content with podcasts and videos as well as self-evaluation activities, forums to create social interaction between a geographically dispersed audience, and gaming elements, among other types of resources. Despite the availability of different learning sources, many online courses focus on teaching processes that are based on passive linear information transfer, in part because the dynamics of learning within networked environments and their effect on pedagogy have not been entirely appropriated by course designers.

While not always exemplified in online courses, technology has allowed people to connect and create a variety of communities that serve different purposes such as social connection, information, professional support, among others. According to Richardson (2012), technology has created a reality where:

Content is everywhere: Knowledge is readily available through digital means from many sources.

Teachers are everywhere: Emphasis is on location of the expertise to support individual learning.

Learning is personalized: Learning is tailored to cater for individual differences and learners have many opportunities to learn what they are interested in learning. 
Networks are the new classroom: Learning is formed around people with common interest and differences in expertise.

Learning is everywhere: Learning doesn't require a bounded type of organization. Learning occurs more in small groups.

Within such networks, a student can take many different paths and obtain different results. How can instructional designers, teachers and other stakeholders design environments that can be freely explored but that also lead to the desired instructional objectives and also motivate students? Combining these elements together and creating a seamless experience that results in effective learning is a challenge for training designers.

This paper suggests that a given network has a specific information architecture (nodes and connections), based on a pedagogical approach and technical considerations, where it is possible to track resource use through data analytics techniques. In particular, this research focuses on sequences followed by students within the network and includes other measures that are expected to help relate those sequences to cognitive load and learning outcomes.

\section{Emerging Pedagogies}

Before dealing with a short review of networked learning, it is important to clarify that as new technology-based strategies are used, we need a set of criteria to define an effective pedagogy. As James and Pollard (2011) propose, an effective pedagogy is one that results in a beneficial synergy among economic productivity, social cohesion and personal development. Gros (2016) suggests that emerging pedagogies are characterized by the following ten characteristics:

1. Supporting lifelong learning.

2. Supporting ecologies of learning.

3. Using different forms of knowledge.

4. Using technology as mindtools.

5. Changing the traditional role of teachers and students.

6. Integrating self-regulation, co-regulation and social share regulation.

7. Promoting deep learning tasks.

8. Making practices transparent.

9. Using socioconstructivist pedagogies.

10. Using new forms of assessment.

Not all of these characteristics are entirely new in online instruction. Current best practices include faculty training to take a leadership or coaching role, more adequate forms of assessment, among others (King, 2014). Emerging pedagogies should incorporate current and innovative practices and combine different tools in learning networks in order to create an improved technology-enhanced experience. 


\subsection{Networked Learning}

There are a variety of definitions of networked learning (Sloep, 2016). Its central aspect is the use of technology to enable connectivity between learners, learners and teachers, and learners and resources (Dohn et al., 2018). Carvalho and Goodyear (2014) conclude that a good definition of networked learning should:

1. Allow one to identify the boundaries of a learning network.

2. Use vocabulary not often used in formal education.

3. Consider both social and technological aspects.

4. Both individual and collective action contributes to the overall dynamics.

These characteristics are a more macro view of the traditional representation of a network as mentioned at the beginning of this paper: a set of nodes (resources, activities, etc.) and connections that allow for a variety of paths, based on emerging pedagogical approaches. Apart from connectivity, networked learning can create ecosystems that merge seamlessly with different types of environments for continuous learning, uses technology as collaborative or individual tools, goes beyond content in order to foster deep learning, makes use of distributed expertise and social agreements, and allows individuals and groups to construct knowledge. Traditional assessment may not be enough to evaluate student strategies and their impact inside this type of environment, so complementary tools are used in this research for evaluation purposes by focusing on the nodes, the interactions between them and the sequences that emerge from the system.

The design of a networked learning space requires dealing with three aspects (Sloep, 2016). The epistemic aspect is related to the activities that the learners have to perform to reach certain outcomes. The social aspect refers to connecting people with shared goals. Finally, the set aspect refers to the technological and narrative context that enables interactions.

Implicit in the design of networked environments is an ideal path, the one the designer has considered when creating the digital learning space. Individual sequences depart from it in response to different personal criteria such as information search, environment exploration, plan following, etc. It is hypothesized that sequence analysis can help measure the degree of departure from that ideal sequence and combined with other dimensions such as traditional assessment, can produce a measure of information entropy, assumed here to be the level of confusion experienced by the student within a learning space.

There are several definitions of entropy in information systems. Regardless of the use of this concept, it is frequently described as the level of uncertainty, either of a signal or more generally of a specific information structure (Baddeley et al., 2008). That level of uncertainty has been found in other digital environments and has been evidenced through the kind of sequences that appear in sequential analysis (Sanchez-Lozano, 2010).

Students frequently experience increased cognitive load in discovery or exploratory environments because of their weak knowledge of foundation concepts (Kirschner et al., 2006). Data analytic methods can be used to explore log data and identify sources of cognitive load, for example through increased levels of interactions with the system resources (Zheng, 
2017). It is expected that those interactions will reveal specific sequence patterns that can be analyzed and related to other constructs.

\section{Research Description}

A postgraduate course on user experience is being studied to better understand the dynamics of networked learning using sequence analysis. Current online courses focus on information transfer although they naturally include a certain level of practice. Interviews with students have shown that some of them encounter difficulties adapting to the dynamics of online training and feel that they are unable to reach their desired proficiency level. Reasons include anxiety with the new learning format, low self-efficacy and time management.

\subsection{Course Design}

As mentioned above there are three aspects that must be taken into consideration when designing for networked learning: epistemic, social and set design (Sloep, 2016).

From the epistemic point of view, the current iteration of the course is based on two main aspects. Content is organized using a flipped-classroom approach, but it is complemented using synchronous sessions, which are in turn based on cognitive apprenticeship. Rather than explanatory sessions, each synchronous meeting is used to develop part of a real company scenario as a team (social set) and not a demonstration. During these sessions, roles switch from student/teacher to coach/team. Based on what students bring to the session, a working relationship is possible because a baseline has been established. Students know that if they do not prepare, their involvement not only will be limited but will cause delays to the team. The case study also serves as the set dimension of the learning space.

Note that cognitive apprenticeship is taken here as "a focus on cognitive skills and process rather than only physical skills development, the use of skills in varied contexts rather than only the context of their use, and the use of structured rather than entirely naturalistic opportunities for skill development" (Cakmakci et al., 2020, p. 294). The information structure of the course is coded in weekly vectors of nodes within Moodle, but also integrate other digital environments such as Slack.

As mentioned before, resources, activities, forum interventions, and all other events are considered to be the network nodes. Emerging connections are the resulting paths that can be visualized using process mining. These along with resource use will help identify cognitive load and information entropy levels.

\subsection{Preliminary Findings}

Preliminary sequence analysis has shown significantly different interaction patterns within the same environment, suggesting the possibility that these patterns are expressions of learning strategies, cognitive load or other underlying processes. Figure 1 shows some of these paths where it is possible to see the strong differences between students' paths, with one outlier corresponding to the teacher. It is possible to see an 'average-length' sequence but also longer and shorter ones. Different colors show different activities performed by each student. 


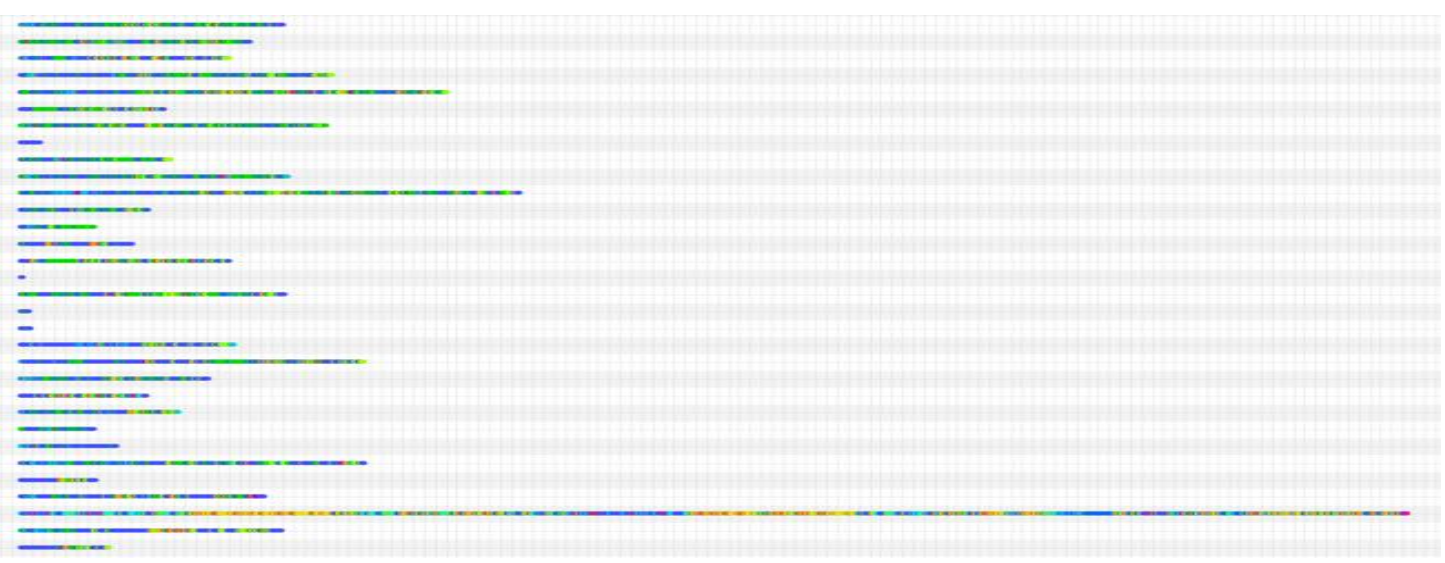

A cluster analysis shows the presence of distinct groups as seen in figure 2. Even though the analysis is based on activity, there seem to be other dimensions that would explain the clusters. One of the objectives of the study is to identify these additional dimensions.

Figure 2: Preliminary Cluster Analysis

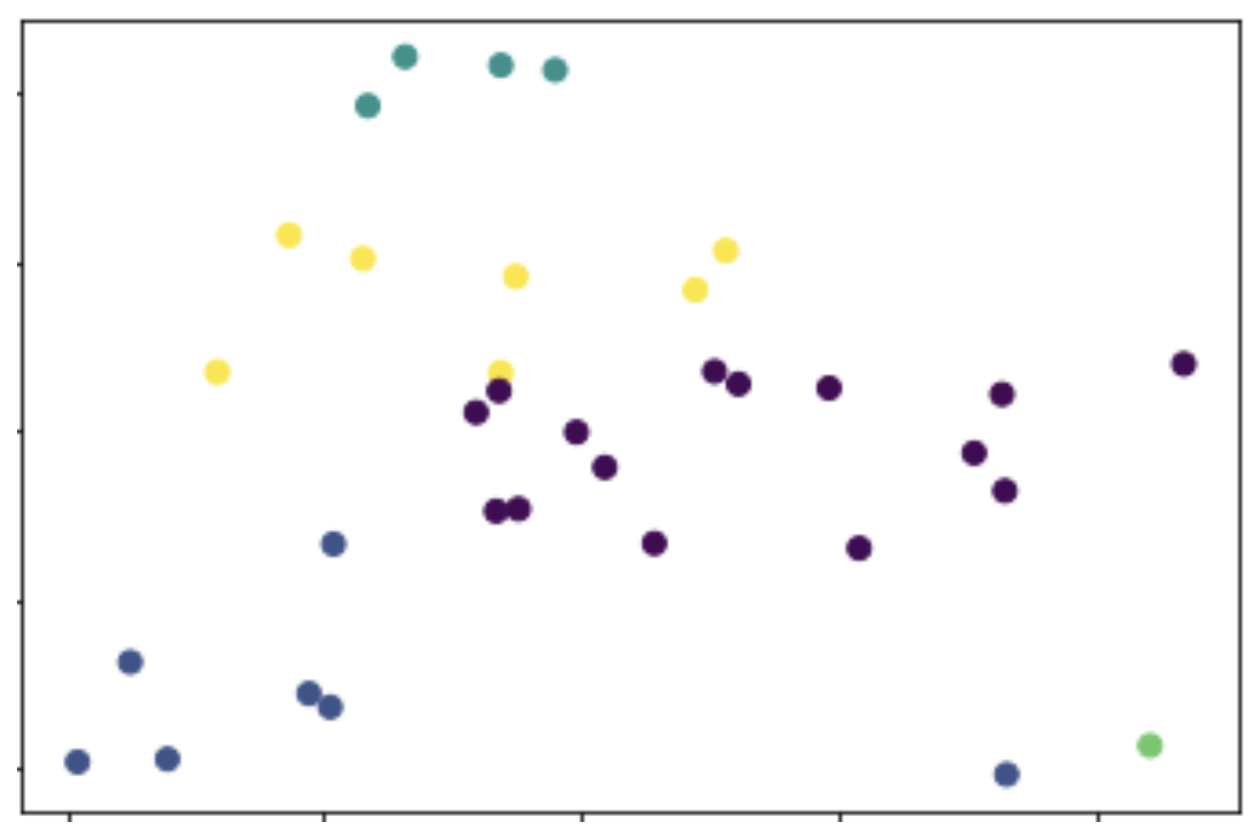

Process mining also shows differences among students and variations between weeks, which cannot be explained using only platform logs (figures 3 and 4). 


\section{ARERL}

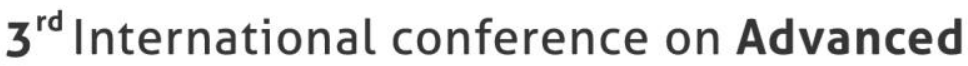 Research in Education, Teaching \& Learning}

Figure 3: Sample Sequence Variation

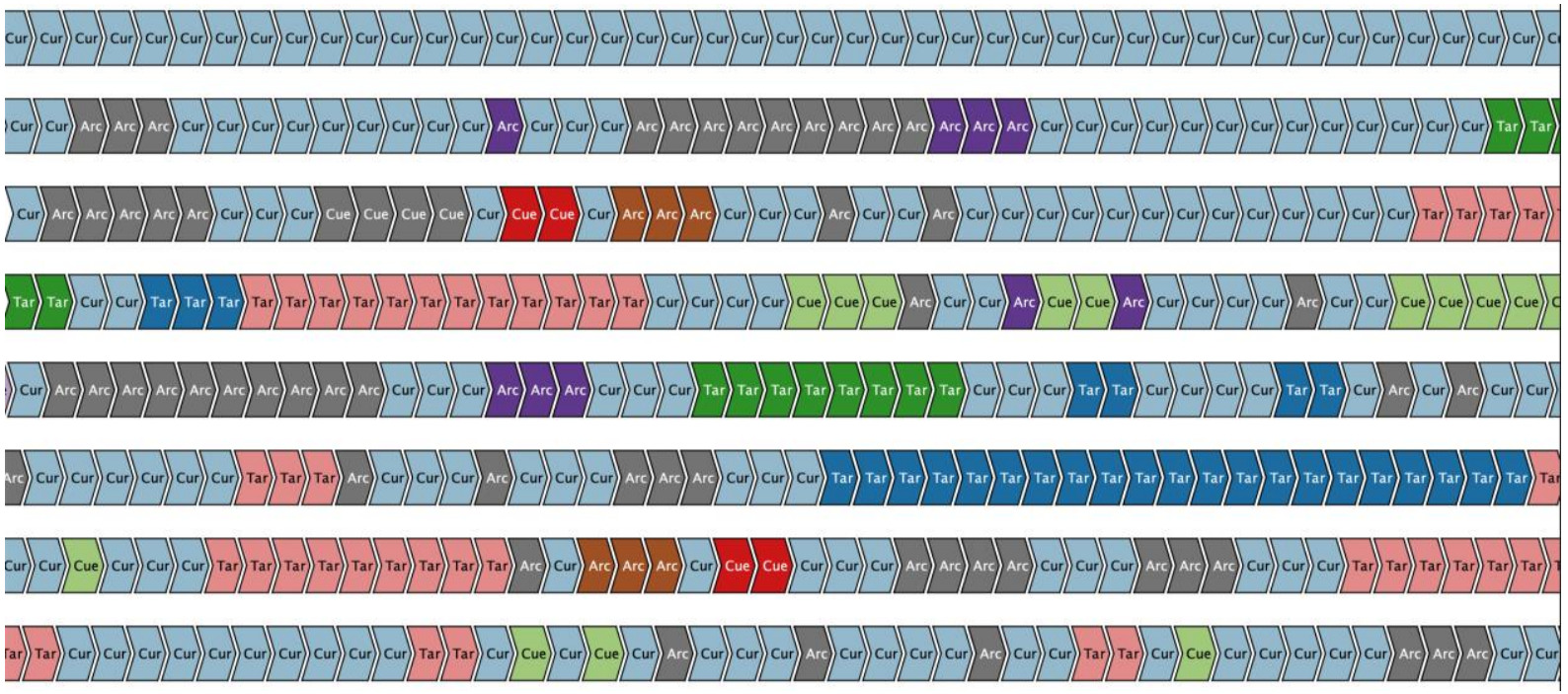

Figure 4: Process Mining

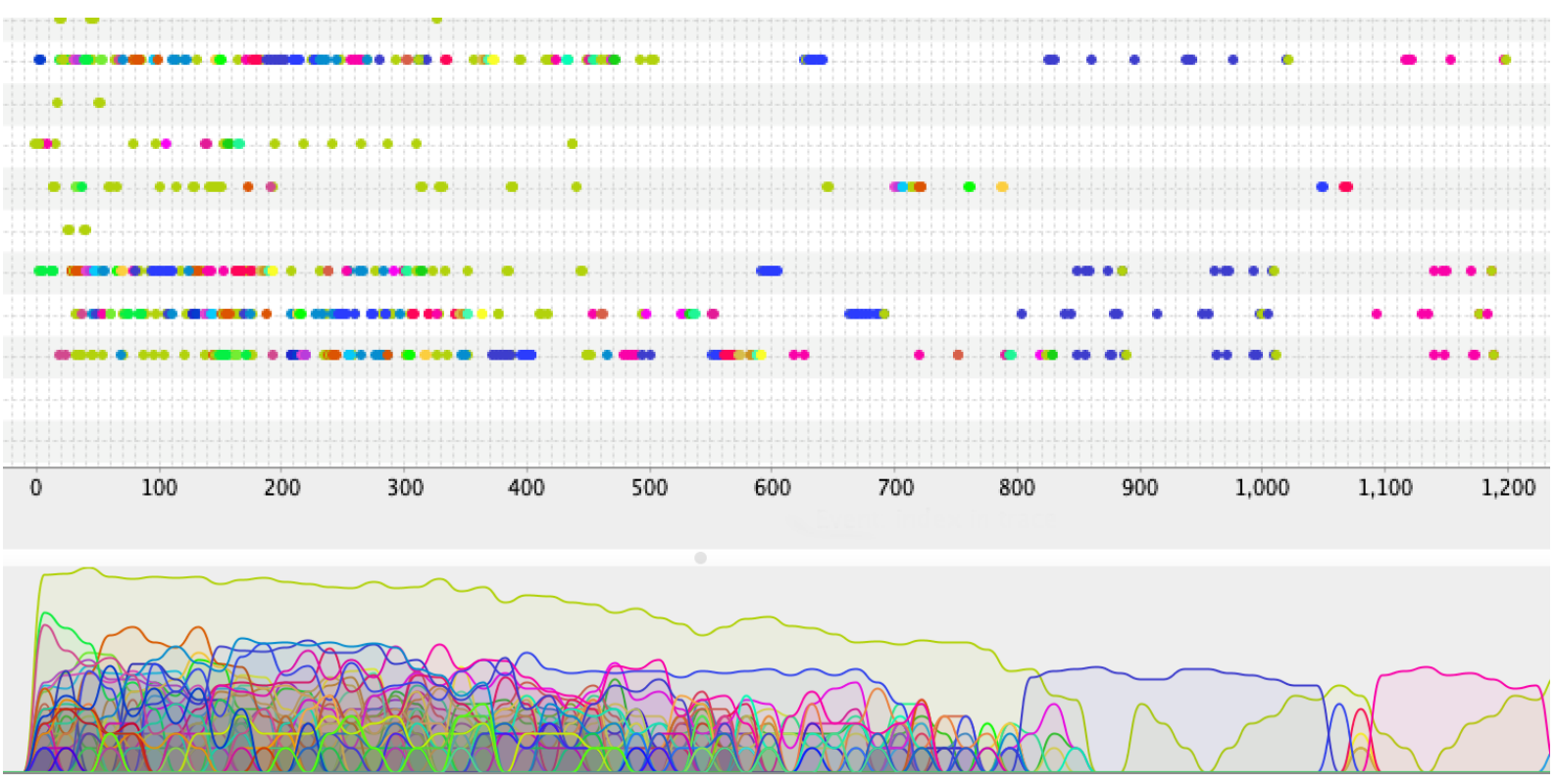

\subsection{Methods}

Action research has been chosen as the most suitable methodology since it is important that all stakeholders collaborate in order to improve the course as new findings emerge. It involves students, teachers and researchers, critically analyzing different data sources and improving processes in a cyclical manner, and drawing practical, usable conclusions that can be integrated and further expanded through evaluation and reflection (Putman \& Rock, 2016). It 


\section{AREIL}

\section{$3^{\text {rd }}$ International conference on Advanced Research in Education, Teaching \& Learning}

is also believed that it might be helpful for students to visualize the dynamics of the strategies they are using, which are not always evident.

The research presented in this paper explores course effectiveness in delivering learning outcomes and retention as measured with traditional assessment tools, student strategies and processes as evidenced by sequential analysis, and additional dimensions such as social interactions on different channels during course delivery.

The hypothesis is that student paths in the network are a reflection of personal strategy as well as resource suitability and usefulness but can also be a reflection of high cognitive load, information entropy, and/or emotional difficulties.

In order to test this hypothesis, sequential analysis or process mining is being carried out every week during the entire 10-week course using the activity logs created by Moodle and analyzing them using PRom. These logs will be combined with other measures such as Slack logs, social interaction and performance measures. In addition, a dashboard using different Python data analytics libraries such as Pandas and Scikit-Learn has been built to track, analyze and get insights from aggregated aspects such as level of activity and session duration, as seen in figure 5 .

\section{Figure 5: Analytics Dashboard}

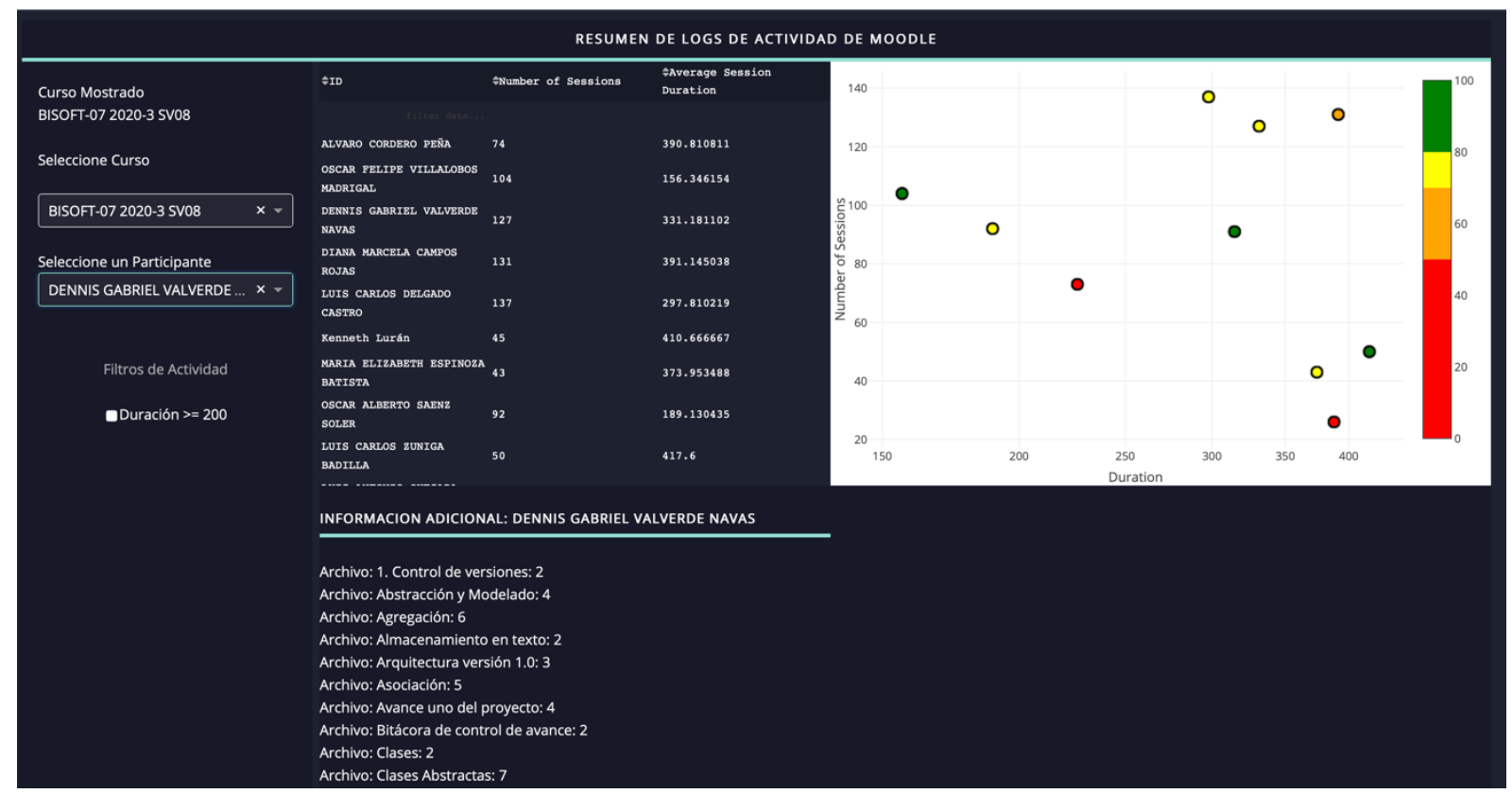

Sequential analysis will be combined with sentiment analysis, text analysis, teamwork scores and traditional evaluations to explore possible associations between sequences and student performance (individual and team) as well as traditional evaluation measures such as quizzes and projects. This part of the research addresses the epistemic and social sets of the course from the point of view of structure but does not consider the emerging interactions 
within the synchronic sessions based on cognitive apprenticeship. For this reason, sessions will be recorded and analysed using qualitative analysis software.

\section{Final Thoughts}

There has always been interest in online courses, in particular specific guidelines that can be used to better design them, achieve learning objectives and improve retention. Despite the large amount of research on this topic, there are still many aspects of online learning dynamics that have not been completely identified.

By using an action research methodology, along with sequence analysis and data analytic methods, it is expected that significant amounts of information will lead to an important improvement in course dynamics and identification of learning strategies and student action patterns inside networked environments.

Beyond the immediate expected benefits of this approach for the course itself, this project aims to contribute more generally to the literature in online courses and networked learning spaces.

\section{References}

Baddeley, R., Hancock, P., \& Földiák, P. (Eds.). (2008). Information theory and the brain. Cambridge University Press.

Cakmakci, G., Aydeniz, M., Brown, A., \& Makokha, J. M. (2020). Situated Cognition and Cognitive Apprenticeship Learning. In Science Education in Theory and Practice (pp. 293310). Springer, Cham.

Carvalho, L., \& Goodyear, P. (2014). The architecture of productive learning networks. Routledge.

Dohn, N. B., Cranmer, S., Sime, J. A., de Laat, M., \& Ryberg, T. (Eds.). (2018). Networked Learning: Reflections and Challenges. Springer.

Gros, B. (2016). The dialogue between emerging pedagogies and emerging technologies. In The Future of Ubiquitous Learning (pp. 3- 23). Springer, Berlin, Heidelberg.

James, M., \& Pollard, A. (2011). TLRP's ten principles for effective pedagogy: rationale, development, evidence, argument and impact. Research Papers in Education, 26(3), 275-328.

King, E., \& Alperstein, N. (2014). Best practices in online program development: Teaching and learning in higher education. Routledge.

Kirschner, P., Sweller, J., \& Clark, R. (2006). Why minimal guidance during instruction does not work: An analysis of the failure of constructivist, discovery, problem-based, experiential, and inquiry-based teaching. Educational Psychologist, 41(2), 75-86.

Putman, S. M., \& Rock, T. (2016). Action research: Using strategic inquiry to improve teaching and learning. Sage Publications. 


\section{ARERL}

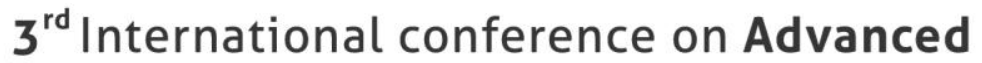

Research in Education, Teaching \& Learning

Richardson, W. (2012). Why School: How education must change when learning and information are everywhere. Ted Conferences.

Sánchez Lozano, J. C. (2010). Distributed information resources and embodied cognition in software application training: interaction patterns in online environments and digital games (Doctoral dissertation, Concordia University).

Sloep, P. B. (2016). Design for networked learning. In The Future of Ubiquitous Learning (pp. 41-58). Springer, Berlin, Heidelberg.

Zheng, R. Z. (Ed.). (2017). Cognitive load measurement and application: a theoretical framework for meaningful research and practice. Routledge. 